\title{
Development of PIAA Complex Mask Coronagraphs for large aperture ground-based telescopes
}

\author{
Kevin Newman ${ }^{\mathrm{a}, \mathrm{b} *}$, Dan Sirbu ${ }^{\mathrm{b}}$, Ruslan Belikov ${ }^{\mathrm{b}}$, Olivier Guyon ${ }^{\mathrm{a}}$ \\ ${ }^{a}$ University of Arizona, Tucson, AZ \\ ${ }^{\mathrm{b}}$ NASA Ames Research Center, Moffett Field, CA
}

\begin{abstract}
The Phase Induced Amplitude Apodization Complex Mask Coronagraph (PIAACMC) is an architecture for directly observing extrasolar planets, and can achieve performance near the theoretical limits for any direct-detection instrument. The PIAACMC architecture includes aspheric PIAA optics, and a complex phase-shifting focal plane mask that provides a pi phase shift to a portion of the on-axis starlight. The phase-shifted starlight is forced to interfere destructively with the un-shifted starlight, causing the starlight to be eliminated, and allowing a region for high-contrast imaging near the star.

The PIAACMC architecture can be designed for segmented and obscured apertures, so it is particularly well suited for ground-based observing with the next generation of large telescopes. There will be unique scientific opportunities for directly observing Earth-like planets around nearby low-mass stars. We will discuss design strategies for adapting PIAACMC for the next generation of large ground-based telescopes, and present progress on the development of the focal plane mask technology. We also present simulations of wavefront control with PIAACMC, and suggest directions to apply the coronagraph architecture to future telescopes.
\end{abstract}

Keywords: Focal plane mask, Coronagraph, PIAA, PIAACMC

\section{INTRODUCTION}

The direct imaging of extrasolar planets is a challenging task that requires precise instrumentation. Many different coronagraph architectures have been proposed to resolve the small angular separation and obtain the deep contrast ratio that is required to observe extrasolar planets. The Phase-Induced Amplitude Apodization (PIAA) [1] technique has emerged as one of the highest performing coronagraph architectures, particularly for its high throughput and small inner working angle. The PIAA technique uses aspheric optics to apodize the light beam in the pupil plane, which allows for a small inner working angle with almost no loss of planet light. Guyon et al. then proposed a modification to the PIAA design which incorporates a phase shifting focal plane mask [2]. The resulting PIAA Complex Mask Coronagraph (PIAACMC) approaches the theoretical performance limit of any direct imaging technique. In addition to its particularly high performance, PIAACMC is also useful because it can be designed for centrally obscured and segmented apertures [3], which is necessary to be compatible with next-generation telescopes such as the Giant Magellan Telescope (GMT), the Thirty Meter Telescope (TMT), and the European Extremely Large Telescope (EELT).

The aspheric PIAA optics have been well characterized and developed over the past few years [4, 5, 6], but a remaining task is to develop and demonstrate the phase-shifting complex focal plane mask. For a monochromatic coronagraph, the mask can be as simple as a half-wave plate sized to provide a phase shift to half of the starlight. However, creating a mask that can operate achromatically in broadband is more challenging. Newman et al. [7] outlined the design process for an achromatic phase-shifting focal plane mask, and discussed one method for manufacturing. The mask manufacturing process is not perfect, and results in mask profile errors that translate into phase errors, which degrade the performance of the coronagraph. In this paper, we take a deeper look at the mask manufacturing errors and how they affect coronagraph performance. In particular, we will study the effects of errors in the mask zone depths. The mask manufacturing process is still under development, and we expect the residual errors to improve. However, we can

Advances in Optical and Mechanical Technologies for Telescopes and Instrumentation II, edited by Ramón Navarro, James H. Burge, Proc. of SPIE Vol. 9912, 99126L

(C) 2016 SPIE · CCC code: $0277-786 X / 16 / \$ 18 \cdot$ doi: $10.1117 / 12.2232164$ 
compensate for some errors by using wavefront control. We will analyze the ability of wavefront control to correct for mask errors and recover coronagraph performance. Kern et al. [8] produced a similar analysis for the Wide-field Infrared Survey Telescope (WFIRST) PIAACMC design, and found that wavefront control was more than capable of correcting for any errors in the fabricated focal plane mask. We expand that analysis to other PIAACMC designs for other telescope pupils, and attempt to make a generalized statement about using wavefront control with PIAACMC.

* newman.kevin.e@gmail.com

\section{EXOPLANET SCIENCE POTENTIAL}

There is a wealth of potential exoplanet discovery and science space that is yet to be explored. Observing exoplanets in reflected light (visible and near-infrared spectrum), in comparison to thermal emission, has a more challenging contrast ratio, but the smaller requirement for telescope aperture size is more practical. For this reason, we will focus on directly observing in reflected light. The most scientifically interesting scenario is to study planets that could be potentially habitable, and are therefore located in the habitable zone of their host star. Figure 1 shows the star-planet separation angle and contrast that would be necessary to observe an Earth-like planet with reflected light in the habitable zone around our nearest stars, out to 20 parsecs distance. Beyond this distance, direct imaging becomes impractical with current methods. There is no guarantee that these theoretical planets exist, but we can use this analysis to determine what type of tools we need to develop to push the boundaries of exoplanet science.

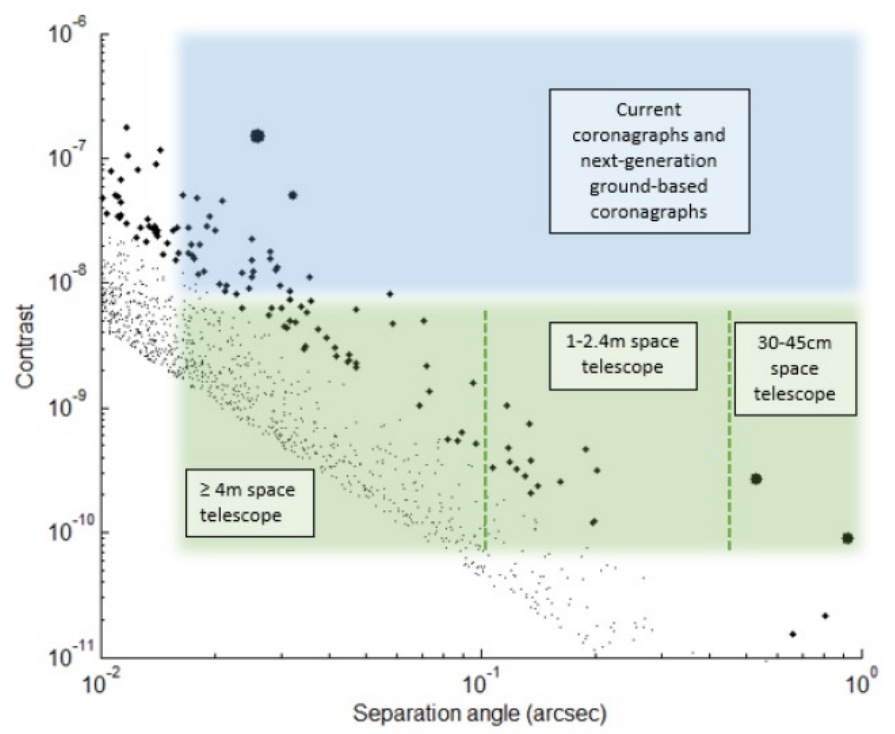

Figure 1: Contrast and separation angle required to directly observe a hypothetical Earth-size exoplanet with reflected light at quadrature in the habitable zone around nearby stars.

In general, the planets towards the lower left corner of Figure 1 are more challenging to observe, and the planets towards the upper right corner of the graph are less challenging. A planet located around a given star that is located close to Earth can be observed with a relatively large angular separation. If the same star and planet are moved further away from Earth, the angular separation will be smaller and more challenging to resolve. Another important characteristic is the host star's stellar type. For a faint star, such as an M-dwarf, the habitable zone is located relatively close to the star. Therefore a habitable planet would have a small angular separation, but it would be able to reflect a greater portion of the starlight, and could be observed with an easier contrast $\left(10^{-7}\right)$. The habitable zone around a bright star is located relatively far from the star, so the angular separation of a habitable planet is greater, but the contrast is more challenging 
$\left(10^{-9}-10^{-11}\right)$. Future coronagraphs and systems that are currently in development will enable imaging for many of the potential planets. The potential exoplanet discoveries can be grouped into categories based on the location and size of the telescope that would be required to observe them.

Because of the effects of the atmosphere, coronagraphs are usually incapable of observing at deeper than $10^{-8}$ contrast from the ground. In space, contrast is only limited by the design of the system and the angular size of the star. Therefore all planets that require deeper than $10^{-8}$ contrast should be observed from space. However, it is more practical to construct a large telescope aperture on the ground. While space-based telescopes have been limited to relatively small aperture sizes, such as the Hubble Space Telescope $(2.4 \mathrm{~m}$ aperture) and the James Webb Space Telescope $(6.5 \mathrm{~m}$ aperture), the next generation of ground-based telescopes are designed to have primary mirrors that are tens of meters in diameter. The TMT will have a $30 \mathrm{~m}$ aperture, the GMT will have a $24.5 \mathrm{~m}$ aperture, and the EELT will have a $39 \mathrm{~m}$ aperture. The large apertures can easily resolve the angular separation between many nearby stars and their habitable zones, however, there are relatively few potential Earth-size planets with a contrast that can be achieved from the ground. However, there is a unique science potential for these telescopes to observe Earth- size planets around the habitable zones of nearby M-dwarf stars. M-dwarfs provide a particularly favorable situation for observing in the habitable zone with reflected light because the stars are relatively dim, so the habitable zone is located closer to the star; this causes the contrast ratio in the habitable zone to be more favorable $\left(10^{-7}\right)$ and within the limits of ground-based observing.

From space, there is great potential to observe Earth-like planets around many nearby stars. Some of the planets would require an aperture size that is unprecedented for space telescopes, but there is no theoretical barrier to the discovery of these planets. The spaced-based observations are separated into three categories in Figure 1 based on the size of the telescope aperture that would be required to observe them, assuming that we observe at $550 \mathrm{~nm}$ wavelength with a coronagraph with a $2 \lambda / \mathrm{D}$ IWA. The potential planets around our nearest star, Alpha Centauri, are particularly lowhanging fruit for exoplanet science, as they can be observed with only a $30-45 \mathrm{~cm}$ space telescope. The Alpha Centauri Exoplanet Satellite (ACESat) mission has been proposed to directly observe around these stars [9, 10]. For observing potential Jupiter-size planets in the habitable zone around nearby stars, the contrast is multiplied by a factor of 121 . This multiplication factor is based on the square of the relative surface area for a Jupiter-size planet compared with an Earthsize planet. The resulting planets, shown in Figure 2, are much easier to observe. In this case, there are many potential Jupiter-size planets that can be observed from the ground with current and next-generation telescopes. For comparison, the large planets that have already been observed with thermal emission are shown with red dots in Figure 2 .

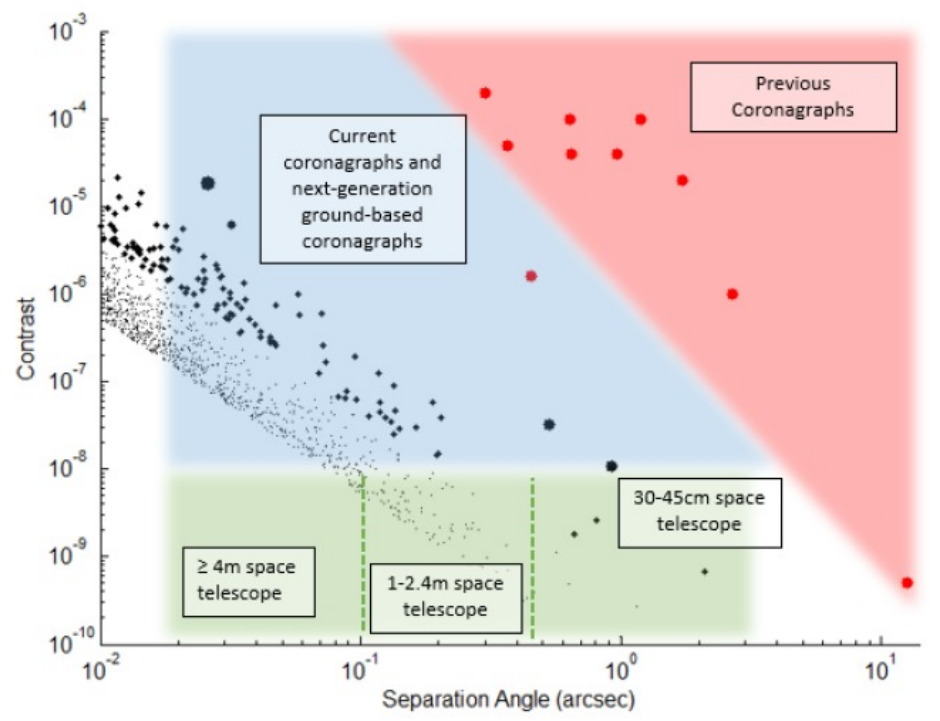

Figure 2: Contrast and separation angle required to directly observe a hypothetical Jupiter-size exoplanet with reflected light at quadrature in the habitable zone around nearby stars. The giant planets that have been observed in thermal emission with previous coronagraphs are shown with red dots for comparison. 
In Section 3, we will discuss the development of technology that will enable the detection of these potential planets. We will demonstrate that we have developed the tools necessary to expand the boundaries of exoplanet science, especially for coronagraphs that can be applied to the next-generation of ground-based telescopes. We can develop all of the components necessary to observe the planets using existing and proven technology.

\section{DEVELOPMENT OF PIAA COMPLEX MASK CORONAGRAPH}

A standard PIAA coronagraph uses pupil apodization produced with PIAA optics to achieve a high contrast PSF while maintaining full throughput of planet light. An opaque disk focal plane mask is used to block the central core of the stellar PSF, and a Lyot stop is used to limit diffraction from the edges of the focal plane mask. The PIAACMC is an improvement on the PIAA coronagraph architecture, where the opaque disk focal plane mask is replaced with a partially transmissive phase-shifting focal plane mask. This technique combines the full throughput of PIAA with the low inner working angle (IWA) that is possible with a phase mask. The IWA is the angular separation from the host star at which the coronagraph can achieve 50\% throughput of the planet light. This is an important metric for coronagraph performance because it describes the minimum angular separation at which the coronagraph can get sufficient light from the planet to identify the planet and perform scientific characterization.

The phase mask of a PIAACMC design only needs to provide a phase shift to a portion of the stellar PSF, and therefore the mask can be significantly smaller than an opaque mask in a conventional PIAA design, which needs to block the entire stellar PSF. The smaller mask allows for a smaller IWA. PIAA optics for a conventional PIAA coronagraph generally have strong apodization that approaches zero intensity towards the edge of the pupil. This technique limits diffraction ringing around the stellar PSF in the focal plane. With a phase mask, the system can accept more diffraction ringing in the focal plane because the mask can be designed to compensate with destructive interference. This trade-off allows the PIAA optics in a PIAACMC system to have a milder profile, which has the practical advantage of being easier to manufacture.

The phase mask in a PIAACMC system allows more design flexibility, which is important for compatibility with complex apertures, such as segmented and centrally obscured apertures. The primary challenge with complicated apertures is to control the diffraction, and the phase mask is an adjustable tool that can be designed for any aperture shape. PIAACMC performs favorably in comparison to other coronagraph architectures, especially considering the high throughput for planet light. In some cases, PIAACMC can approach the theoretical performance limit for any direct imaging instrument [2].

PIAA optics have been thoroughly developed and demonstrated, so the only remaining piece of technology that needs to be developed in order for PIAACMC to be successful is the phase-shifting focal plane mask. For observing in monochromatic light, the focal plane mask can be as simple as a circular phase plate. However, for the mask to work in broadband, the design and manufacturing of the mask becomes more challenging. We have developed strategies for the design and manufacturing of the mask [7], and in this section we will explore the use of wavefront control with PIAACMC, specifically to correct for mask manufacturing errors. Our goal is to develop the final piece of technology to enable PIAACMC to be applied to current and future telescopes and provide high-performance exoplanet science.

A typical PIAACMC apodization profile is shown in Figure 3a. The focal plane mask is circular with a 60 micron outer diameter, which is divided into ten circularly symmetric ring-shaped zones, as shown in Figure 3b. The focal plane mask design is shown in Figure 3c. The mask is designed for a $10 \%$ bandwidth centered at $580 \mathrm{~nm}$. The depth of each zone is optimized to provide the phase shift necessary to achieve deep contrast over the entire bandwidth as described in [7]. We aim for an average broadband raw contrast (PSF surface brightness) on the order of $10^{-7}$, which is suitable for exoplanet science with ground-based telescopes, and is also suitable for space-based telescopes that are interested in observing circumstellar disks and Jupiter-size planets. Before wavefront control, the system has $4.9 * 10^{-7}$ average contrast between 2 and $6 \lambda / \mathrm{D}$. The mask could be scaled laterally as a function of radius, and axially as a function of depth in order to 
accommodate another range of wavelengths. The PIAACMC architecture is certainly capable of achieving deeper contrast and smaller IWA than these designs, but the goal of this section is to demonstrate wavefront control, so particularly high contrast is not necessary. Contrast levels on the order of $10^{-7}$ are already ambitious goals for groundbased observatories.

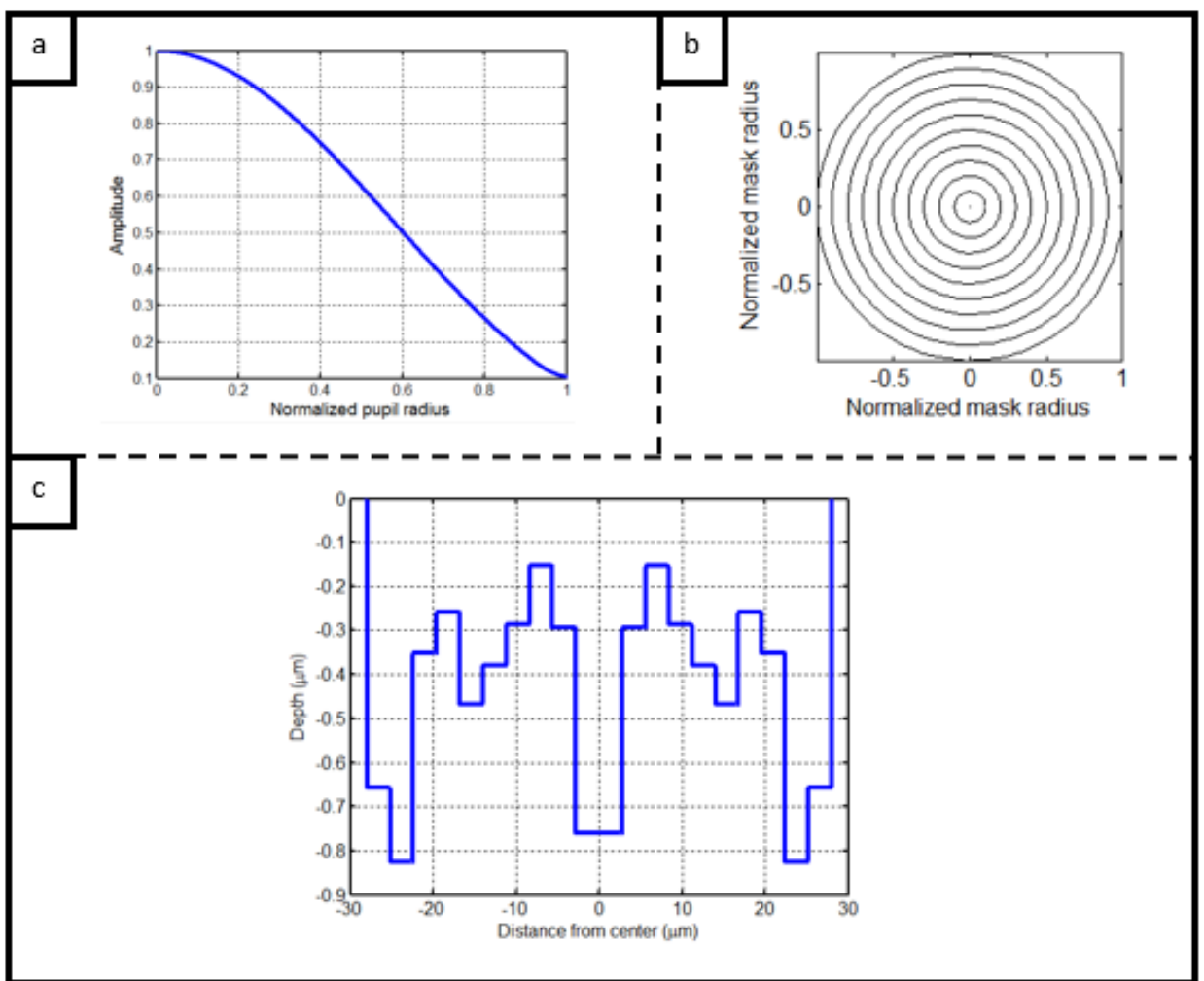

Figure 3: Radially symmetric apodization profile of the modified PIAA optics at the Ames Coronagraph Laboratory (a) and structure of the focal plane mask design (b). A cross section profile of the focal plane mask (c).

A variety of PIAACMC focal plane masks have been manufactured at the Stanford Nanofabrication Facility and the NASA Jet Propulsion Laboratory, Microdevices Laboratory. The manufacturing process for such small and precise devices is not a trivial task, yet the labs have achieved good results from manufacturing $[7,8]$. However, there are still residual manufacturing errors, the most common of which is a difference between the designed and manufactured depth for a particular zone in the mask. Results from one particular manufacturing session were shown in [7], along with the resulting degredation of corongraph performance. In this section we will more broadly characterize how these typical mask manufacturing errors affect the performance of the coronagraph system, and how well we can compensate for these errors with the use of wavefront control. Starting with the mask design, we simulated adding random amounts of error to the depths of each zone in the mask, and calculated the resulting coronagraph performance. Results are shown with the red dots in Figure 4. 


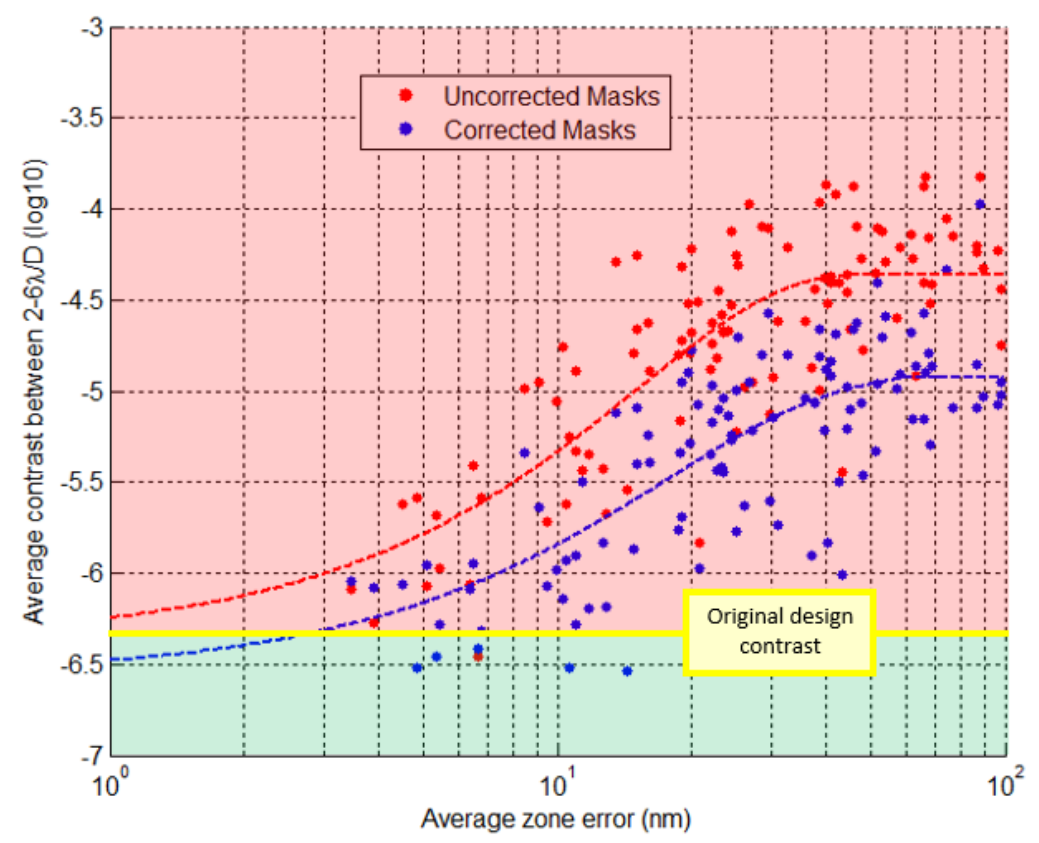

Figure 4: Contrast of a PIAACMC design with random manufacturing errors added to the focal plane mask (red dots). The contrast can be improved, up to a certain level, with the use of wavefront control (blue dots).

We simulated 150 trials of the mask with independent randomly selected errors added to the depth of each zone. The errors were selected from a uniformly distributed range between $-\mathrm{N}$ and $+\mathrm{N}$ for different values of $\mathrm{N}$ between 0 and $200 \mathrm{~nm}$. Errors assigned to different zones within a mask were uncorrelated. On the x-axis of Figure 4, we show the average of the absolute value of all depth errors in the mask. The y-axis in Figure 4 shows the resulting average contrast within a specified dark region. The dark region spans radially from 2 to $6 \lambda / \mathrm{D}$, and azimuthally from $+90^{\circ}$ to $-90^{\circ}$. The region does not include the area within $0.5 \lambda / \mathrm{D}$ in the $\mathrm{x}$-direction to avoid a situation where the deformable mirror $(\mathrm{DM})$ is attempting to correct regions that are separated by a $180^{\circ}$ angle. The individual contrast values are fitted with a fourthorder polynomial that reaches a stable peak as the average zone error increases. For zero error, the system performance starts with the originally designed contrast, and as the error increases it follows a steep curve of worsening contrast before it peaks at $4.3 * 10^{-5}$. There is a high degree of variability in the system performance, even for masks with similar amounts of error. However, we can identify a few important trends in the system performance. Without any strategy for performance correction, the average mask error would need to remain less than $5 \mathrm{~nm}$ in order to achieve within an order of magnitude of the original design contrast. Once the average error in the mask has reached $40 \mathrm{~nm}$, the performance reaches a plateau, which suggests that any additional error in the system has little effect on the performance.

To correct for the effect of errors in the mask zone heights, we implemented an electric field conjugation (EFC) wavefront control algorithm. In this investigation the mask errors in the PIAACMC focal plane create an aberrated field in the final focal plane. For these simulations, we assume that the rest of the coronagraph system does not contribute any aberrations, and that we have perfect knowledge of the electric field in the final focal plane. The EFC algorithm uses the spectrum of the aberrated electric field in the final focal plane and computes a corresponding DM setting that corrects these aberrations at a plane which is conjugate to the PIAA exit pupil (where the DM is located). EFC is a model-based wavefront correction technique and requires a linear model of the effect of each DM actuator poke (change in actuator height) in the correction region of the final image plane. For these simulations, we model the DM that is in use at the ACE laboratory, which is a Boston Micromachines DM with a grid of 32 × 32 actuators. A regularized least-squares solution computes the required DM actuator heights (DM settings) to conjugate these aberrations. DM settings are computed for the central wavelength $(580 \mathrm{~nm})$ and maintained as the wavelength band is extended to broadband. The WFC algorithm does not always reach an ideal solution, so we eliminated WFC results where the algorithm had diverged to a bad solution, which usually occurred because the regularization parameter search was not exhaustive enough. Many of these cases were clearly identified because the best contrast was at the outer edge of the design bandwidth; this should 
not occur for an ideal WFC solution because the system is corrected at the center wavelength. We also eliminated a few WFC solutions that resulted in a contrast worse than $1 * 10^{-3.5}$, because these solutions were clearly outliers and not representative of the WFC algorithm performance. In total we eliminated approximately $25 \%$ of the WFC data.

The remaining WFC simulations, shown as blue dots in Figure 4, have a similar behavior compared with the uncorrected masks. However, the contrast follows a shallower curve as a function of mask zone error, and reaches a peak at $1.2^{*} 10^{-5}$. For some masks with an average zone error of less than $15 \mathrm{~nm}$, WFC can achieve contrast that is better than the original design. With any greater amount of error, wavefront control cannot achieve the original design contrast. Therefore the average mask depth error should be kept to less than $15 \mathrm{~nm}$ in order to achieve the full science potential of the coronagraph design. Our manufacturing results [7] achieved an average mask depth error of $14 \mathrm{~nm}$, so these wavefront control simulations suggest that in many cases, the manufacturing accuracy may already be good enough to achieve full system performance. For errors greater than $50 \mathrm{~nm}$, there is little difference in the achievable contrast, but WFC can still improve the contrast by an order of magnitude in comparison to an uncorrected system. These results were obtained with a particular implementation of EFC that has been established at the ACE testbed for use with PIAA systems. It is possible that another implementation of EFC or a different WFC strategy altogether could produce better results for correcting mask performance.

\section{CONCLUSIONS AND FUTURE WORK}

The PIAA Complex Mask Coronagraph is a powerful and versatile coronagraph architecture which can be designed for any arbitrary telescope aperture, and can approach the theoretical performance limit for any direct detection system. The two core pieces of technology needed for a PIAACMC system are the aspheric PIAA optics and a phase shifting focal plane mask. The PIAA optics have been thoroughly developed and demonstrated. We have developed a design strategy for the phase shifting focal plane mask, and have demonstrated manufacturing capabilities that can create the masks with high precision. The simulations in this paper demonstrate that wavefront control is capable of compensating for residual focal plane mask manufacturing errors, and that current manufacturing capabilities will not limit the performance of a PIAACMC system. We presented an example mask design which is valuable for observing Jupiter-size planets with a ground-based telescope, and other PIAACMC systems can be designed to observe Earth-size planets with a space-based telescope. All of the capabilities are currently in place for the PIAACMC coronagraph architecture to be a significant contributor in the field of direct exoplanet imaging. This work was supported by a NASA Space Technology Research Fellowship. 


\section{REFERENCES}

[1] O. Guyon, "Phase-Induced Amplitude Apodization of Telescope Pupils for Extrasolar Terrestrial Planet Imaging", A\&A v.404, p. 379-387 (2003).

[2] O. Guyon, F. Martinache, R. Belikov, R. Soummer, "High Performance PIAA Coronagraphy with Complex Amplitude Focal Plane Masks," ApJ, 190-2, pp. 220-232 (2010).

[3] O. Guyon, P. Hinz, E. Cady, R. Belikov, F. Martinache, "High Performance Lyot and PIAA Coronagraphy for Arbitrarily Shaped Telescope Apertures," ApJ, 780-171 (2013).

[4] P. Lawson, R. Belikov,W. Cash, M. Clampin, T. Glassman, O. Guyon, J. Kasdin, B. Kern, R. Lyon, D. Mawet, D. Moody, R. Samuele, E. Serabyn, D. Sirbu, J. Trauger, "Survey of Experimental Results in High-Contrast Imaging for Future Exoplanet Missions," Proc. SPIE, 8864-50 (2013).

[5] R. Belikov, E. Bendek, T. Greene, O. Guyon, J. Lozi, D. Lynch, K. Newman, E. Pluzhnik, G. Schneider, D. Tenerelli, S. Thomas, F. Witteborn, "EXCEDE Technology Development II: Demonstration of High Contrast at 1.2 L/D and preliminary broadband results," Proc. SPIE, 8864-31 (2013).

[6] D. Sirbu, S. Thomas, R. Belikov, J. Lozi, E. Bendek, E. Pluzhnik, D. Lynch, T. Hix, P. Zell, G. Schneider, "EXCEDE technology development III: demonstration of polychromatic contrast in vacuum at $1.2 \mathrm{~L} / \mathrm{D}$," Proc. SPIE, 9605-18 (2015).

[7] K. Newman, J. Conway, R. Belikov, O. Guyon, "Focal plane phase masks for PIAA: design and manufacturing," PASP 128, 963 (2016).

[8] B. Kern, O. Guyon, R. Belikov, D. Wilson, R. Muller, E. Sidick, K. Balasubramanian, J. Krist, I. Poberezhskiy, H. Tang, "PIAACMC Mask Fabrication, Characterization, and Modeling for WFIRST-AFTA," JATIS (to be published).

[9] R. Belikov, E. Bendek, S. Thomas, et al., Proc. SPIE, 9605 (2015).

[10]E. Bendek, R. Belikov, J. Lozi, et al., Proc. SPIE, 9605 (2015). 